\title{
Bilobar Colorectal Liver Metastases: Challenges and Opportunities
}

\author{
Matthew E. B. Dixon, MD, FACS ${ }^{1}$ (D), and Niraj J. Gusani, MD, MS, FACS ${ }^{2}$ \\ ${ }^{1}$ Program for Liver, Pancreas, and Foregut Tumors, Division of Surgical Oncology, Penn State College of Medicine, \\ Hershey, PA; ${ }^{2}$ Section of Surgical Oncology, Baptist MD Anderson Cancer Center, Jacksonville, FL
}

The boundaries of possibility in hepatic surgery continue to expand. Refinements in surgical and anesthetic techniques, deeper understanding of hepatic anatomy, development of new technologies and strategies for hepatic transection, and improvements in postoperative care all have contributed to making hepatic surgery safer while also broadening the universe of patients who might benefit from this therapy. As surgeons versed in the principles of modern hepatic surgery through dedicated training programs at high-volume centers become more widely disseminated, surgical options can be offered to more and more patients who need them. Patients with colorectal liver metastases (CRLM) represent an important group who stand to gain from these advancements.

Historically, CRLM has been associated with a poor outcome and a 5-year overall survival rate as low as 5.3\%. ${ }^{1}$ Surgical resection with or without ablation of liver metastases allows selected CRLM patients to achieve 5 -year overall survival rates well above $50 \% .^{2}$ Not surprisingly, hepatectomy for CRLM, when feasible, has become the standard of care in this population for more than two decades. ${ }^{3}$

Despite these data, enthusiasm is tempered by the fact that only $25 \%$ to $30 \%$ of patients with CRLM are deemed resectable at presentation. ${ }^{3-5}$ Therefore, devising strategies to improve resectability for these patients carries a very high priority. Bilobar distribution of CRLM represents a

(C) Society of Surgical Oncology 2021

First Received: 25 November 2020

Accepted: 28 November 2020;

Published Online: 3 January 2021

N. J. Gusani, MD, MS, FACS

e-mail: niraj.gusani@gmail.com challenging clinical management scenario for patients previously deemed unresectable who may be given the opportunity to benefit from surgery, with many approaches devised to tackle this problem.

The surgical options for bilobar CRLM currently include anatomic hepatectomy, one-stage parenchymalsparing hepatectomy, and traditional two-stage hepatectomy (TSH) with or without portal vein embolization (PVE), as well as associated liver partition and portal vein ligation for staged hepatectomy (ALPPS) and liver transplantation. Any one of these approaches for patients with CRLM relies heavily on precise and accurate assessment of resectability based on the size, location, and distribution of the tumors and their proximity to the portal and hepatic vein branches, all with the goal of eventually preserving an adequate future liver remnant (FLR).

The need to perform a right hepatectomy with additional left-sided resections (as is the case in most patients with multiple bilobar CRLM) exposes patients to a high risk of post-hepatectomy liver failure. Therefore, many patients will require hypertrophy of the FLR before resection via PVE. Furthermore, there is evidence that metastases in the FLR can grow more rapidly than in the non-tumor-containing hepatic parenchyma in the FLR after PVE, so these lesions should ideally be resected before a PVE. ${ }^{6,7}$ Therefore, a staged approach using TSH can be a powerful strategy for removing all CRLM while leaving an adequate FLR in patients who would otherwise be unresectable with a single operation. ${ }^{8}$

In this issue of Annals of Surgical Oncology, Chavez et al. ${ }^{9}$ examines the presentation, management, and outcomes of patients who completed TSH in a cohort in five major hepatobiliary surgery centers from across the United States. From December 2000 to March 2016, 196 patients with bilobar CRLM were identified who had completed a 
TSH. Notably, the average is only two to three patients per center each year from some of the largest and most experienced liver surgery centers in the country. This illustrates how few patients will successfully complete this surgical approach. Despite this, the Chavez et al. ${ }^{9}$ study represents one of the largest cohorts investigating outcomes of TSH for CRLM to date, adding important data to the literature.

The results of this study are largely similar to those of prior studies in terms of perioperative outcomes and overall survival, with a 5-year overall survival rate of $44 \%$ in this series compared with $42 \%$ to $51 \%$ in prior studies. ${ }^{4,10}$ Notably, the median follow-up period for this cohort was only 28 months, so to date, the data are not mature enough to help us understand long-term survival, nor was the dataset adequately powered to analyze survival in a multivariate comparison.

This analysis leaves unanswered a very important question: what proportion of patients who underwent the first stage of planned TSH did not complete the second stage? Going back even further in the timeline, what percentage of patients with extensive liver metastases who were deemed potentially resectable and underwent chemotherapy upfront did not have a good enough response to move forward with any resection strategy?

These data were not tracked in the report by Chavez et al., ${ }^{9}$ whose study included only patients who completed the second stage of TSH. In prior studies, 25 to $31 \%$ of the patients did not proceed to the second stage of planned $\mathrm{TSH}^{4,6,10}$ In the current series with more modern chemotherapy options, would we see similar results? For 61 to $94 \%$ of patients unable to proceed to the second stage, the most common reason historically has been disease progression. What was the reason in the current cohort?

This leads to the question whether chemotherapy should be administered between stages of TSH. The rationale for administration of chemotherapy after the first hepatectomy procedure includes evidence that growth factors inducing hypertrophy of parenchymal cells after liver resection and portal embolization also may have the potential to stimulate tumor cells. ${ }^{70-12}$ This notion must be tempered with the concern for further hepatotoxicity from chemotherapy as more chemotherapy is administered.

In the current series, $60.5 \%$ of the patients underwent chemotherapy between stages of TSH, with an interstage interval of $4 \pm 3.1$ months. This is comparable with the series described by Wicherts et al., ${ }^{10}$ in which $78 \%$ of the patients underwent chemotherapy between the stages of $\mathrm{TSH}$, with a mean interstage interval of 4.2 months.

Importantly, in the current study, the patients who had an interstage interval shorter than 6 months had a better overall survival after TSH. This difference likely was multifactorial, representing the patients in the group with an interval longer than 6 months who had worse disease biology and a tumor burden harder to control with systemic chemotherapy. It also could be a reflection of hepatic toxicity from prolonged systemic chemotherapy and its contribution to adverse perioperative outcomes.

As noted earlier, an inadequate FLR is a principal concern when patients are considered for TSH. ${ }^{13}$ Our most important tool for manipulating the volume of the FLR still is PVE (alternatively, portal vein ligation). It is heavily used in the management of bilobar CRLM, particularly when a right or extended right hepatectomy is planned at the second stage.

In the current series, only $65 \%$ of the patients underwent a PVE, a lower rate than in prior studies although $75.5 \%$ of the patients underwent a major hepatectomy at the second stage. ${ }^{4,6,10}$

Accurate calculation of the FLR before major hepatectomy is an important part of surgical planning, and welldefined volume cutoffs have been reported previously. Of the patients for whom PVE was omitted, 35\% likely had an adequate FLR volume, which begs the question why they needed a TSH in the first place. It also is possible that the longer median interstage interval allowed for more hypertrophy of the FLR after the first resection, obviating the need for PVE.

Approximately $15 \%$ of patients will have an inadequate FLR after PVE. A notable adjunctive option for these patients is hepatic vein embolization (HVE) in combination with PVE, which can safely be added to gain even more hypertrophy of the FLR. Laurent et al. ${ }^{14}$ recently reported a series of 73 patients who underwent PVE either with or without simultaneous HVE. Simultaneous PVE and HVE resulted in a hypertrophy of $16.98 \%$ versus only $8.46 \%$ with PVE alone. For patients found to have an inadequate FLR, HVE may help to increase the size of the FLR further to a sufficient volume and should be considered more widely to help more patients reach the second stage of TSH.

The application of ALPPS remains controversial. Prior studies have shown that although ALPPS enabled an adequate FLR to be reached in a shorter time, this came at the cost of higher morbidity and mortality rates than with TSH. Furthermore, although not shown in the current series, the most common reason for patients not undergoing the second stage of TSH generally is disease progression, not an inadequate FLR. ${ }^{4,6,10}$ The LIGRO trial, which compared TSH and ALPPS for the management of patients with CRLM, showed a higher rate for completion of planned liver surgery with ALPPS (84\% of the patients completed ALPPS vs $54 \%$ of the patients completing TSH). ${ }^{15}$ The reason patients did not proceed to the second stage of TSH in the LIGRO trial was almost always an inadequate FLR, per the trial's primary end point. Newer adjuncts such as 
HVE affording even greater increases in volume of the FLR may further reduce the rate of patients failing to proceed to the second stage of TSH while making ALPPS (with its incumbent higher morbidity) less frequently needed.

On a system level, an important issue to consider is underutilization of liver surgery in general for CRLM. As mentioned previously, TSH was used in the current series for only two or three patients per center annually. Although this partly resulted from the fact that few patients are candidates for TSH, it also may reflect under-referral of bilobar CRLM patients to hepatobiliary surgeons for evaluation by medical oncologists and other associated providers early in the course of disease.

In a survey of medical oncologists in the state of Michigan, Krell et al. ${ }^{16}$ found wide variation in referral of patients to a hepatobiliary surgeon for consideration of surgical resection and identified both bilobar disease and large tumor size as the most commonly perceived contraindications to surgical resection of CRLM. Defining resectability is paramount in multimodal treatment planning and relies on detailed understanding of hepatic surgical anatomy, which is possible only if a hepatobiliary surgeon is involved upfront in evaluation of all patients with CRLM. Therefore, TSH often may not be used because patients with bilobar CRLM are under-referred for evaluation. We strongly reiterate that all such patients should be referred to hepatobiliary surgeons upfront for assessment of surgical options.

Patients with bilobar CRLM remain a challenging group to evaluate and treat. Currently, TSH remains an important, safe surgical management strategy for these patients, with acceptable postoperative outcomes and represents an opportunity to offer potentially curative treatment. However, it seldom is used, as demonstrated in the current study. As more data emerge for the TSH approach, all patients with CRLM should be referred to hepatobiliary surgeons for accurate assessment of resectability and consideration of this technique.

\section{REFERENCES}

1. Beppu T, Miyamoto Y, Sakamoto Y, et al. Chemotherapy and targeted therapy for patients with initially unresectable colorectal liver metastases, focusing on conversion hepatectomy and longterm survival. Ann Surg Oncol. 2014;21(Suppl 3):405-13.

2. Pulitano C, Bodingbauer M, Aldrighetti $\mathrm{L}$, et al. Liver resection for colorectal metastases in presence of extrahepatic disease: results from an international multi-institutional analysis. Ann Surg Oncol. 2011;18:1380-8.

3. Mise Y, Aloia TA, Brudvik KW, et al. Parenchymal-sparing hepatectomy in colorectal liver metastasis improves salvageability and survival. Ann Surg. 2016;263:146-52.

4. Broquet A, Abdalla E, Kopetz S, et al. High survival rate after two-stage resection of advanced colorectal liver metastases: response-based selection and complete resection define outcome. J Clin Oncol. 2011;29:1083-90.

5. Wei A, Jarnagin W. Questioning why more patients with colorectal liver metastases are not referred for metastatectomy. JAMA Surg. 2020. https://doi.org/10.1001/jamasurg.2020.1460.

6. Jaeck D, Oussoultzoglou E, Rosso E, et al. A two-stage hepatectomy procedure combined with portal vein embolization to achieve curative resetion for initially unresectable multiple and biolobar colorectal liver metastases. Ann Surg. 2004;240:1037-49.

7. Elias D, De Baere T, Roche A, et al. During liver regeneration following right portal embolization the growth rate of liver metastases is more rapid than that of the liver parenchyma. $\mathrm{Br} J$ Surg. 1999;86:784-8.

8. Cloyd J, Aloia TA. Hammer versus Swiss Army Knife: developing a strategy for the management of bilobar colorectal liver metastases. Surgery. 2017;162:12-7.

9. Chavez MI, Gholami S, Kim BJ, et al. Two-stage hepatectomy for bilateral colorectal liver metastases: a multi-institutional analysis. Ann Surg Oncol. 2020. https://doi.org/10.1245/s10434020-09459-6.

10. Wicherts DA, Miller R, de Haas RJ, et al. Long-term results of two-stage hepatetomy for irresectable colorectal cancer liver metastases. Ann Surg. 2008;248:994-1005.

11. Picardo A, Karpoff HM, Ng B, Lee J, Brennan MF, Fong Y. Partial hepatecctomy accelerates local tumor growth: potential roles of local cytokine activation. Surgery. 1998;124:57-64.

12. Kokudo N, Tada K, Seki M, et al. Proliferative activity of intrahepatic colorectal metastases after preoperative hemihepatic portal vein embolization. Hepatology. 2001;34:267-72.

13. Dixon M, Cruz J, Sarwani N, Gusani N. The future liver remnant: definition, evaluation, and management. Am Surg. 2020. https://d oi.org/10.1177/0003134820951451.

14. Laurent C, Fernandez B, Marichez A, et al. Radiological simultaneous portohepatic vein embolization (RASPE) before major hepatectomy: a better way to optimize liver hypertrophy compared to portal vein embolization. Ann Surg. 2020;272:199-205.

15. Sandstrom P, Rosok BI, Sparrelid E, et al. ALPPS improves resectability compared with conventional two-stage hepatectomy in patients with advanced colorectal liver metastasis: results from a Scandinavian multicenter randomized controlled trial (LIGRO trial). Ann Surg. 2018;267:833-40.

16. Krell RW, Reames BN, Hendren S, et al. Surgical referral for colorectal lilver metastses: a population-based survey. Ann Surg Oncol. 2015;22:2179-94.

Publisher's Note Springer Nature remains neutral with regard to jurisdictional claims in published maps and institutional affiliations. 